\title{
Cloning and Expression of Bovine Sodium/Glucose Cotransporter SGLT2*
}

\author{
F.-Q. Zhao, T. B. McFadden, E. H. Wall, B. Dong, and Y.-C. Zheng \\ Lactation and Mammary Gland Biology Group, Department of Animal Science, \\ University of Vermont, Burlington, 05405
}

\begin{abstract}
The second member of the $\mathrm{Na}^{+} /$glucose cotransporter family, SGLT2, is a low-affinity active glucose transporter. In humans, it is predominantly located on the apical membrane of the S1 and S2 segments of renal proximal convoluted tubules, and, thus, may be mainly responsible for the reabsorption of D-glucose from the glomerular filtrate. By BLAST searching the GenBank database, we found expressed sequence tag sequences of SGLT2 in the cDNA library of bovine mammary tissues, indicating its expression in bovine mammary gland. To facilitate study of the mechanism of glucose reabsorption in bovine kidneys in maintenance of glucose homeostasis of lactating cows and the potential role of SGLT2 in the mammary gland, we cloned bovine SGLT2 and examined the distribution of its mRNA expression in bovine tissues. The full length mRNA of bSGLT2 is $2275 \mathrm{bp}$, and is predicted to encode a protein of 673 amino acids, with a molecular weight of approximately $73 \mathrm{kDa}$. The deduced amino acid sequence of bovine SGLT2 is $91,90,91$, and $90 \%$ identical to human, rabbit, mouse, and rat SGLT2, respectively, and is 58 and $48 \%$ identical to bovine SGLT1 and SGLT5, respectively. The sequence of bSGLT2 contains several characteristically conserved sodium:solute symporter family signatures. Analysis of current bovine genomic data indicates that the bovine SGLT2 gene may consist of 14 exons. The major in vitro transcription and translation product of bovine SGLT2 cDNA migrated at an apparent molecular weight of $55 \mathrm{kDa}$. The SGLT2 mRNA was detected predominantly in bovine kidney as a 2.3$\mathrm{kb}$ transcript, and at lower levels in all other bovine tissues examined, including the mammary gland, liver, lung, spleen, intestine, and skeletal muscle, as a 3.0$\mathrm{kb}$ transcript. Expression of SGLT2 mRNA in bovine mammary gland increased more than 10 -fold from late pregnancy to early lactation, similar to SGLT1. This
\end{abstract}

Received February 5, 2005.

Accepted April 4, 2005.

Corresponding author: F.-Q. Zhao; e-mail: fzhao@uvm.edu.

*The nucleotide sequence data reported in this paper have been submitted to GenBank with assigned accession number AY208941. indicates that SGLT2 may play a role in milk synthesis in the lactating mammary gland.

(Key words: gene expression, glucose transport, glucose uptake, mammary gland)

Abbreviation key: bSGLT1, bSGLT2, bSGLT5 = bovine solute carrier family 5 (sodium/glucose cotransporter) member 1,2 , and 5 (gene symbols SLC5A1, SLC5A2, and SLC5A5), EST = expressed sequence tag, RACE = rapid amplification of cDNA ends, SGLT = sodium/glucose contransporter, $\mathbf{T M}=$ transmembrane domain.

\section{INTRODUCTION}

The $\mathrm{Na}^{+}$/glucose cotransporters (SGLT) are members of the expanded solute carriers SLC5A family and are predominantly expressed in the brush-border membranes of small intestine and proximal convoluted tubule of the kidney. In these tissues, SGLT mediate an active, sodium-linked glucose transport process against an electrochemical gradient, and play a central role in absorption or reabsorption of glucose and galactose from digesta or from the glomerular filtrate. Thus far, 6 SGLT isoforms (SGLT1 to 6) have been cloned (Wright and Turk, 2004), but only SGLT1 and SGLT2 have been characterized. Only SGLT1 and SGLT5 have been cloned and examined for expression in bovine tissues (Zhao et al., 2005).

Isoform SGLT2 is a low-affinity glucose transporter. It transports $\alpha$-methyl-D-glucoside, a nonmetabolizable glucose model substrate, with an apparent $K_{m}$ of $2 \mathrm{~m} M$, but cannot efficiently transport D-galactose and 3-o-methyl-D-glucose (Wright, 2001). Located predominantly on the apical membrane of the S1 and S2 segments of renal proximal convoluted tubules in human and rat, SGLT2 is presumed to be mainly responsible for the reabsorption of D-glucose from the glomerular filtrate (Wells et al., 1992; Kanai et al., 1994; Wright, 2001). This assumption is further supported by genetic evidence that homozygous and heterozygous nonsense mutations of SGLT2 are associated with autosomal recessive renal glycosuria in human patients (van den Heuvel et al., 2002; Kleta et al., 2004). 
Table 1. Sequences of oligonucleotide primers used for PCR and rapid amplification of cDNA ends.

\begin{tabular}{lll}
\hline Primer name & Type & Sequence \\
\hline bSGLT2-24F & Forward & 5'-AGAATGGAGGAGCACACAGA-3' \\
bSGLT2-1434F & Forward & 5'-GTCTTCGTGTTGGCGCTCTT-3' \\
bSGLT2-1633F & Forward & 5'-TCACCCTCGTGGTCTCACTGT-3' \\
bSGLT2-F1 & Forward & 5'-AGTGCCTGCTCTGGTTCTGT-3' \\
bSGLT2-266R & Reverse & 5'-TGGCCACTGCCGATGTTGCT-3' \\
bSGLT2-1717R & Reverse & 5'-CCTTGCTGTGCCGGAGACT-3' \\
bSGLT2-R1 & Reverse & 5'-CAGCTGGCCAAGGCCTCACA-3' \\
bSGLT2-R2 & Reverse & 5'-ACCTCACAGGGTGTGGCTATG-3' \\
bSGLT2-Probe & Probe & 5'-ACCGCCCATTCCGCGCAAG-3' \\
\hline
\end{tabular}

The kidneys play an essential role in maintenance and regulation of glucose homeostasis in both ruminants and nonruminants. In humans, renal glomeruli generate about $180 \mathrm{~L} / \mathrm{d}$ of filtrate (roughly 70 times the total plasma volume), and about $180 \mathrm{~g}$ of D-glucose are filtered from plasma by the renal corpuscles. Normally, all of the filtered glucose is reabsorbed into blood by the proximal tubules. Undoubtedly, this process occurs on a much larger scale in lactating cows. However, the contribution of renal reabsorption of glucose to body glucose homeostasis in the lactating cow is often overlooked and little relevant information is available.

We used the human cDNA sequence of SGLT2 to BLAST search GenBank and found 2 bovine SGLT2 expressed sequence tag (EST) clones (Accession numbers BE481971, 86\% identity; and BF230764, 91\%) in the USDA Beltsville Agricultural Research Center cDNA library of bovine mammary tissues. This indicated that SGLT2 is expressed in bovine mammary gland. To facilitate study of the mechanism of glucose reabsorption in bovine kidneys, its role in maintenance of glucose homeostasis in lactating cows, and the potential role of SGLT2 in the mammary gland, we cloned bovine SGLT2 and examined the distribution of its mRNA expression in bovine tissues. We report for the first time that SGLT2 is indeed expressed in the bovine mammary gland and that its expression is developmentally regulated in a manner consistent with a possible role in support of lactation.

\section{MATERIALS AND METHODS}

\section{Animals, Tissues, and RNA Isolation}

Unless otherwise specified below, the mammary gland, liver, kidney, lung, spleen, jejunal epithelia, and skeletal muscle tissues were collected from 2 lactating cows, and RNA was isolated from these samples as described previously (Zhao et al., 2005).

\section{Rapid Amplification of cDNA Ends and Cloning of bSGLT2}

The sequences of all primer oligonucleotides used in this study are listed in Table 1.

The $3^{\prime}$ and $5^{\prime}$ sequences of bSGLT2 were obtained by rapid amplification of cDNA ends (RACE) using SMART RACE cDNA amplification kits (Clontech, Palo Alto, CA). The $3^{\prime}$ and 5' RACE-ready first-strand cDNA were synthesized using $1 \mu \mathrm{g}$ of polyadenylated RNA from bovine kidney. The $5^{\prime}$ sequence of bSGLT2 was then amplified using the provided universal primer (UPM, Clontech) and the bSGLT2-specific primer, bSGLT2-266R, which was designed from the bovine SGLT2 EST sequence in GenBank (Accession number AV603260; 89\% identity to human SGLT2 cDNA). The 3 ' sequence of bSGLT2 was amplified using the primers UPM and bSGLT2-1434F designed from another bovine SGLT2 EST sequence (BI538588; 92\% identity to human SGLT2 cDNA). The resulting PCR products were gel-purified, cloned into pCR4-TOPO vector (Invitrogen, Carlsbad, CA) and sequenced using an ABI 377 automated sequencer (Applied Biosystems, Foster City, CA). The sequences of $3^{\prime}$ and $5^{\prime}$ RACE products were verified in at least 3 independent clones.

Bovine full-length cDNA of SGLT2 was amplified from the bovine kidney 5' RACE-ready first-strand cDNA above by PCR using Herculase-enhanced DNA polymerase (Stratagene, La Jolla, CA) with primers bSGLT2-24F and bSGLT2-R1 designed from the $5^{\prime}$ and $3^{\prime}$ untranslated regions of bSGLT2 based on our RACE results. The PCR products were gel-purified and cloned into pCR-BluntII-TOPO vector using Zero Blunt TOPO PCR Cloning kit (Invitrogen) to form bSGLT2/pCRBlunt II-TOPO. The full-length bSGLT2 cDNA was sequenced, verified in at least 3 independent clones, and submitted to GenBank with accession number AY208941.

\section{DNA Sequence Analysis}

The analysis of cDNA sequences was conducted using the computer programs of DNAstar (DNASTAR, Madison, WI), the National Center for Biotechnology Information BLAST site (http://www.ncbi.nlm.nih.gov/ BLAST/), and the SWISS-PROT Scan-Prosite program (http://us.expasy.org/tools/scanprosite/). The multiple sequence alignment was performed with CLUSTAL W (open gap cost 10) (Thompson et al., 1994). The hydropathy plots of bSGLT1 and bSGLT2 were analyzed according to the algorithm of Kyte and Doolittle (1982) using the ProtScale program on the ExPASy proteomics server (http://us.expasy.org/cgi-bin/protscale.pl), with a 19-amino acid window. 


\section{In Vitro Transcription/Translation}

The cDNA of bSGLT2 was excised from the plasmid bSGLT2/pCR-Blunt II-TOPO and subcloned into the vector plasmid pcDNA3.1(-) (Invitrogen) to form plasmid bSGLT2/pcDNA3.1. This plasmid, along with bSGLT1/pcDNA3.1 (Zhao et al., 2005), was transcribed by $\mathrm{T} 7$ polymerase and translated in the presence of $\mathrm{L}$ $\left[{ }^{35} \mathrm{~S}\right]$ methionine (Amersham, Piscataway, NJ) using the TNT Coupled Reticulocyte Lysate System (Promega, Madison, WI). Reactions were performed in the presence or absence of canine microsomal membranes (Promega). The translation products were resolved by $12 \%$ (wt/vol) SDS-PAGE with the addition of $3 M$ urea to the gel and loading buffer to reduce membrane protein aggregation, then imaged using a phosphor-capture screen and ImageQuant software on a Storm PhosphorImager (Molecular Dynamics, Sunnyvale, CA).

\section{Northern Blot Analysis}

Northern blot analysis of bSGLT2 mRNA expression in bovine tissues was carried out as described previously (Zhao et al., 2004). A bovine SGLT2 cDNA probe was generated from the bSGLT2 cDNA clone by PCR using the $5^{\prime}$ primer bSGLT2-F1 and 3' primer bSGLT2R2 (Table 1) to produce a 359-bp product spanning the 3 ' specific region.

\section{Quantitative Reverse Transcription-PCR}

Mammary biopsies were obtained from 12 Holstein cows on $\mathrm{d}-40,-20,-7$, and +7 relative to expected calving ( $\pm 5 \mathrm{~d})$ as described previously (Zhao et al., 2005). Total RNA was isolated from each biopsy sample, treated with deoxyribonuclease (Ambion) to remove contaminating DNA, and treated with RNA Secure (Ambion) to inactivate ribonucleases. Quality of RNA was verified by inspection of the $18 \mathrm{~S}$ and $28 \mathrm{~S}$ rRNA bands after agarose gel electrophoresis. The Stratascript II reverse transcription kit (Invitrogen) was used with oligo dT primers to reverse transcribe $5 \mu \mathrm{g}$ of total RNA into cDNA.

Relative mRNA expression profiles were determined by real-time quantitative PCR using a PE 7700 thermal cycler (TaqMan; Applied Biosystems). The cDNA of each individual cow was diluted 1:4 in nuclease-free water, and $1 \mu \mathrm{L}$ was amplified in a $25-\mu \mathrm{L}$ reaction mix containing $12.5 \mu \mathrm{L}$ of TaqMan $2 \times$ PCR Master Mix (Applied Biosystems). Primers (bSGLT2-1633F and bSGLT2-1717R) and a probe (bSGLT2-Probe) for bSGLT-2 were designed from our cDNA sequence using Primer Express (version 1.5; Applied Biosystems). The PCR reaction started with a 2 -min uracil-glycosylase step at $50^{\circ} \mathrm{C}$ followed by a 10 -min $\mathrm{Taq}$ activation step at $95^{\circ} \mathrm{C}$. Samples were then subjected to 40 cycles of 15 -s denaturation at $95^{\circ} \mathrm{C}$, and 1 -min annealing and extension at $65^{\circ} \mathrm{C}$. Optical data were collected at the end of each extension step, and relative expression of PCR products was determined by comparison with a standard curve. To generate the standard curve, equal amounts of undiluted cDNA of all individual cows were pooled and serial dilutions of the pool were run with the unknowns in each assay. Serial dilutions were: undiluted, 1:5, 1:10, 1:50, 1:100, and a negative control (water). All samples and standards were run in duplicate and mean expression relative to the common pool was determined. All gene expression values were normalized to that of $\beta$-actin in the same sample as previously described (Zhao et al., 2005).

\section{Statistical Analysis}

Statistical analysis was performed using the Mixed Models procedure of SAS (version 8.2; SAS Institute, Inc., Cary, NC). The model included treatment and time as fixed variables and cow as a random variable. Mammary biopsies were included as repeated measures. Comparison of means was performed using Fisher's protected LSD test, and significant differences were declared at $P<0.05$.

\section{RESULTS AND DISCUSSION}

\section{Isolation of the Bovine SGLT2 CDNA by RACE}

We used a RACE strategy to obtain the full-length cDNA sequence of bovine SGLT2 from bovine kidney (Figure 1). The full-length mRNA of bSGLT2 is 2275 bp (GenBank accession number AY208941), and contains an AAUAAA consensus sequence, 16 nucleotides upstream from the polyadenylated tail, which is present in the vast majority of eukaryotic polyadenylation signals (Natalizio et al., 2002). Open reading frame analysis suggested that the bovine SGLT2 protein is composed of 673 amino acids, with a molecular weight of approximately $73 \mathrm{kDa}$.

The sequence of bovine SGLT2 is highly related to that of bovine SGLT1 and SGLT5, which we previously reported (Zhao et al., 2005). The deduced amino acid sequence of bovine SGLT2 is 58 and $48 \%$ identical, or 78 and $66 \%$ conservative to those of bSGLT1 and bSGLT5, respectively (Figure 2). The major differences are in the last loop region between the putative transmembrane domains (TM) 13 and 14 of bSGLT1 (see below) and in the amino-terminal (N-terminal) region (Figure 2).

The deduced amino acid sequence of bovine SGLT2 is $91,90,91$, and $90 \%$ identical, or $94,95,94$, and $93 \%$ 


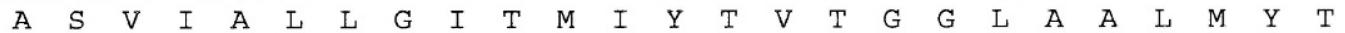
601 GACACGGTGCAGACCTTCGTCATTCTCGCCGGGGCCTTCGTCCTCATGGGTTACGCCTTCCACGAGGTGGGCGGG

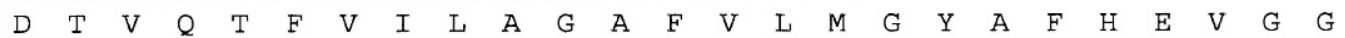
676 TATTCGGGGCTTTTCGACAAATACTTGCGGGCAGTGACGTCCCTGACGGTATCCGAGGATCCGGCCGTGGGCAAC $\begin{array}{llllllllllllllllllllllllllllllllllll}Y & S & G & L & F & D & K & Y & L & R & A & V & T & S & L & T & V & S & E & D & P & A & V & G & N\end{array}$ 751 ATCTCCAGCTCCTGCTATCGACCCCGGCCGGACTCCTACCACCTGCTCCGGGACCCTGTGACGGGGGACCTGCCA $\begin{array}{llllllllllllllllllllllllllllllllll}I & S & S & S & C & Y & R & P & R & P & D & S & Y & H & L & L & R & D & P & V & T & G & D & L & P\end{array}$ TGGCCCGCGCTGCTTCTGGGGCTTACTATCGTGTCCAGCTGGTACTGGTGCAGCGACCAGGTTATAGTGCAGCGC

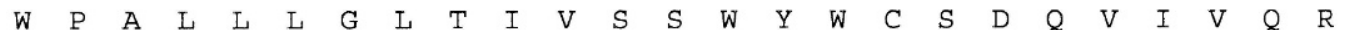
901 TGCCTGGCTGGGAAAAACCTCACCCACATCAAGGCGGGCTGCATCCTGTGCGGCTACTTGAAGCTGATGCCCATG

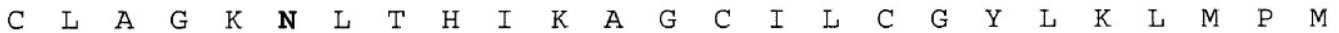
976 TTCCTCATGGTCATGCCTGGAATGATCAGCCGCGTTCTTTACCCGGACGAAGTGGCGTGCGTGGTGCCCGAGGTG $\begin{array}{llllllllllllllllllllllllllllllllll}F & L & M & V & M & P & G & M & I & S & R & V & L & Y & P & D & E & V & A & C & V & V & P & E & V\end{array}$ 1051 TGTAAGCGCGTGTGCGGCACCGAGGTGGGCTGCTCCAATATCGCCTACCCGCGGCTCGTCGTGAAGCTCATGCCC

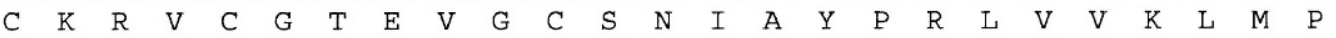
1126 AATGGTCTGCGCGGACTCATGCTGGCGGTCATGCTGGCGGCGCTCATGTCCTCGCTGGCCTCCATCTTCAACAGC $\begin{array}{llllllllllllllllllllllllllllllll}N & G & L & R & G & L & M & L & A & V & M & L & A & A & L & M & S & S & L & A & S & I & F & N & S\end{array}$ 1201 AGCAGCACACTCTTCACCATGGACATCTACACGCGCCTGCGGCCCCGCGCGGGCGACCGCGAGCTGCTGCTAGTA $\begin{array}{llllllllllllllllllllllllll}S & S & T & L & F & T & M & D & I & Y & T & R & L & R & P & R & A & G & D & R & E & L & L & L & V\end{array}$ 1276 GGACGGCTCTGGGTGGTGTTCATCGTGGCCGTGTCGGTGGCCTGGCTGCCCGTGGTGCAGGCGGCGCAGGGCGGG $\begin{array}{lllllllllllllllllllllllllllllllll}G & R & L & W & V & V & F & I & V & A & V & S & V & A & W & L & P & V & V & Q & A & A & Q & G & G\end{array}$ 1351 CAGCTCTTCGATTACATCCAGTCGGTCTCCAGCTACCTGGCGCCGCCGGTGTCGGCCGTCTTCGTGTTGGCGCTC

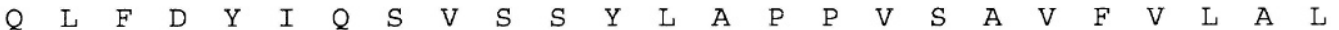
1426 TTCGTGCCCCGCGTCAACGAGAAGGGCGCCTTCTGGGGACTGATCGGGGGCCTGCTGATGGGCCTGGCACGCCTG

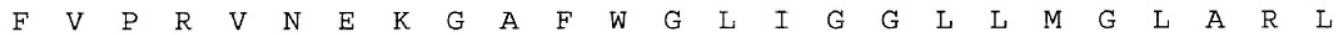
1501. GTTCCCGAGTTCTCCTTCGGCTCCGGCAGCTGCGTGCGCCCCTCCGGGTGCCCGGCTCTCCTCTGCCGCGTCCAC $\begin{array}{llllllllllllllllllllllllllllllllll}V & P & E & F & S & F & G & S & G & S & C & V & R & P & S & G & C & P & A & L & L & C & R & V & H\end{array}$ 1576 TACCTCTACTTCGCCATCCTGCTCTTCGTCTGCTCCGGCCTCCTCACCCTCGTGGTCTCACTGTGCACACCGCCC $\begin{array}{lllllllllllllllllllllllllllllll}Y & L & Y & F & A & I & L & L & F & V & C & S & G & L & L & T & L & V & V & S & L & C & T & P & P\end{array}$ 1651. ATTCCGCGCAAGCACCTCTACCGCCTGGTTTTCAGTCTCCGGCACAGCAAGGAAGAGAGGGAGGACCTGGATGCT $\begin{array}{lllllllllllllllllllllllllll}I & \mathrm{P} & \mathrm{R} & \mathrm{K} & \mathrm{H} & \mathrm{L} & \mathrm{Y} & \mathrm{R} & \mathrm{L} & \mathrm{V} & \mathrm{F} & \mathrm{S} & \mathrm{L} & \mathrm{R} & \mathrm{H} & \mathrm{S} & \mathrm{K} & \mathrm{E} & \mathrm{E} & \mathrm{R} & \mathrm{E} & \mathrm{D} & \mathrm{L} & \mathrm{D} & \mathrm{A}\end{array}$ 1726 GAGGAGCTAGAAGGTCCAACCGCAGCCCCCGTGCAGAACGGGCGCCCTGAGCACGCAGTGGAGATGGAGGCGCCC $\begin{array}{llllllllllllllllllllllllll}E & E & L & E & G & P & T & A & A & P & V & Q & N & G & R & P & E & H & A & V & E & M & E & A & P\end{array}$ 1801 CCGCCCCCAAGGCCAGGCCTGTTGCGGCAGTGCCTGCTCTGGTTCTGTGGCGTGAGCAGGGGTGGGGTGGGCAGC $\begin{array}{llllllllllllllllllllllllllllll}P & P & P & R & P & G & L & L & R & Q & C & L & L & W & F & C & G & V & S & R & G & G & V & G & S\end{array}$ 1876 CCCCAACGCCCTACCCAGGAGGAGACGACTGCTGCAGCCAGGCGGCTGGAGGACATCAGTGAGGACCCACGCTGG $\begin{array}{lllllllllllllllllllllllllllllllllll}P & Q & R & P & T & Q & E & E & T & T & A & A & A & R & R & L & E & D & I & S & E & D & P & R & W\end{array}$ 1951 GCCCGCGTGGTCAACCTCAACGCCCTGCTCATGATGGCCGTGGCCACATTCCTCTGGGGCTTTTATGCCTGAggC $\begin{array}{llllllllllllllllllllllllllllllllll}A & R & V & V & N & L & N & A & L & L & M & M & A & V & A & T & F & L & W & G & F & Y & A & *\end{array}$

2026 ccactgcattggacaccctgagccacagccttagatgagtgggggtggggagccagcggcggtgagaagggcctg 2101 gggccagagagtagaggggaggccccgggacccctactctctgccttgtttctgcctgggacccagtccatagcc 2176 acaccctgtgaggccttggccagctggccactgtagttcccctaagaacaataaagctgccttgcctagtcaaa 2251 aaaaaaaaaa

Figure 1. Full-length cDNA and deduced amino acid sequence of bovine sodium/glucose cotransporter 2 (SGLT2). In the cDNA sequence, lowercase letters represent the $5^{\prime}$ and $3^{\prime}$ untranslated regions, and uppercase letters represent the coding region. The predicted amino acid sequence is shown in uppercase letters beneath the coding sequence. The predicted N-glycosylation sites are shown in bold face, and the AAUAAA consensus sequence is underlined. The nucleotide sequence of bovine SGLT2 mRNA has been submitted to the GenBank nucleotide database with accession number AY208941. 


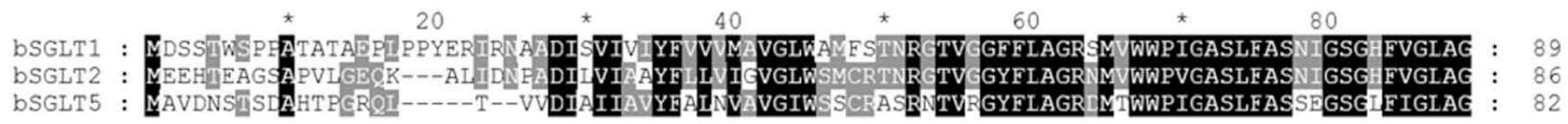

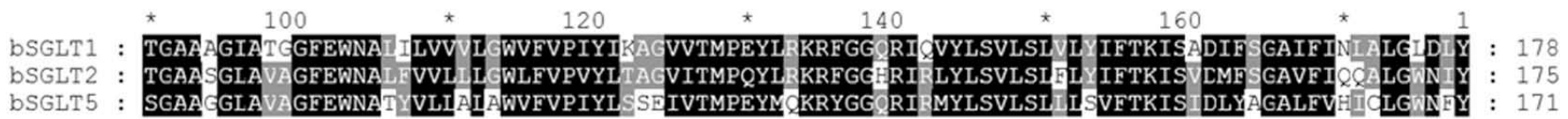
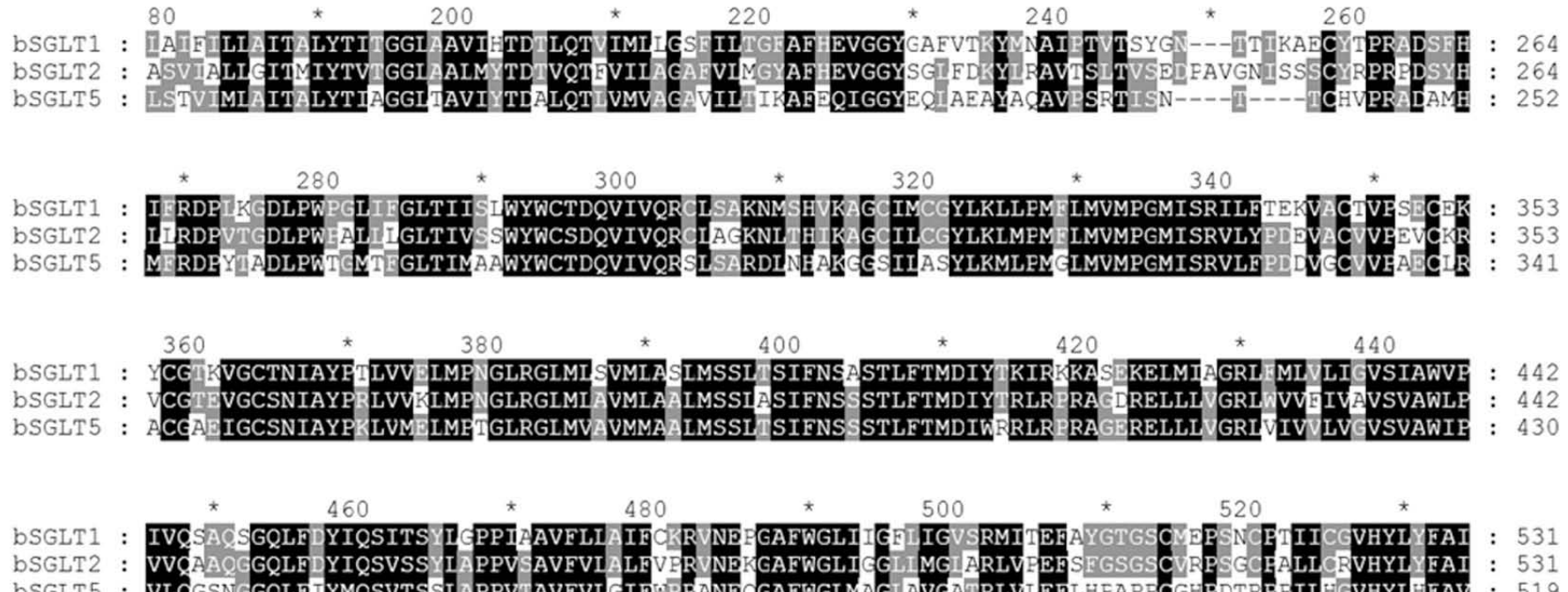

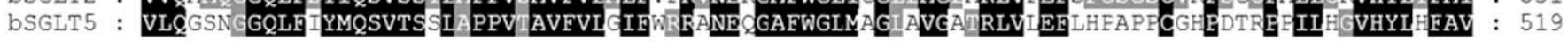
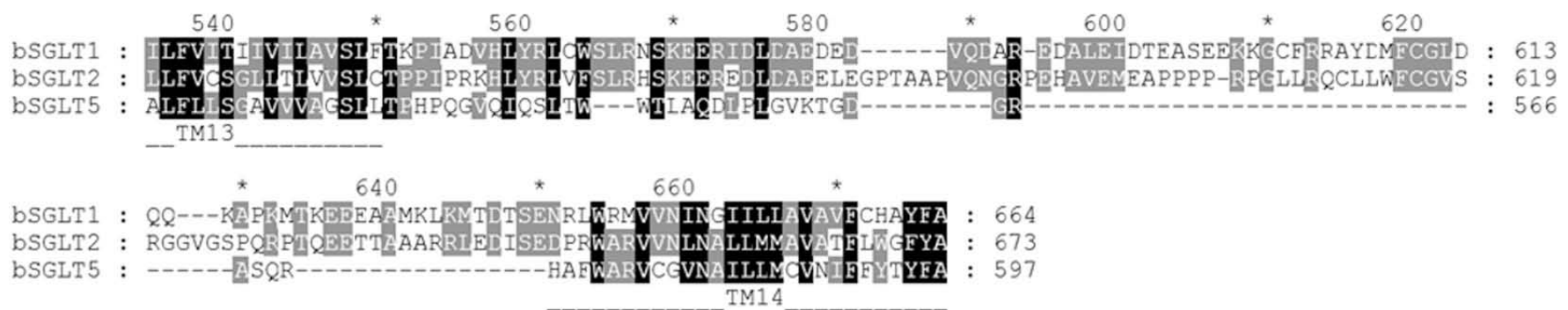

Figure 2. Multiple sequence alignment of the deduced amino acid sequence of bovine sodium/glucose cotransporter 2 (SGLT2) with the sequences of other known members of bovine SGLT family: bSGLT1 (GenBank accession no. AF508807) and bSGLT5 (AY514442). The positions of presumed transmembrane helices (TM13 and 14) in SGLT1 are shown by the numbered dashed lines below the sequence alignments. Residues highlighted by black shading represent absolutely conserved amino acid; residues with gray shading indicate 2 conserved residues at that position.

conservative to human (accession number NM_003041), rabbit (M84020), mouse (NM_133254), and rat (NM_022590) SGLT2 (Figure 3). The major difference between species is also located in the most divergent region among bovine SGLT.

\section{Sequence Analysis of Bovine SGLT2}

The hydropathy plot analysis of bovine SGLT2 by ProtScale revealed a similar distribution of hydrophobic segments as in bSGLT1 (Zhao et al., 2005) in the majority of the sequence (Figure 4). However, a striking difference between the 2 transporters exists in the Cterminal region, where bSGLT2 may have one additional TM in the region corresponding to the long cytoplasmic loop between TM 13 and 14 in SGLT1 (Figures 2 and 4). This is inconsistent with the presumed tertiary structure of SGLT members, which are typically comprised of 14 hydrophobic and presumed TM regions (Turk and Wright, 1997; Wright and Turk, 2004). Thus, bSGLT2 may have 15 membrane-spanning regions vs. 14 in bSGLT1. 

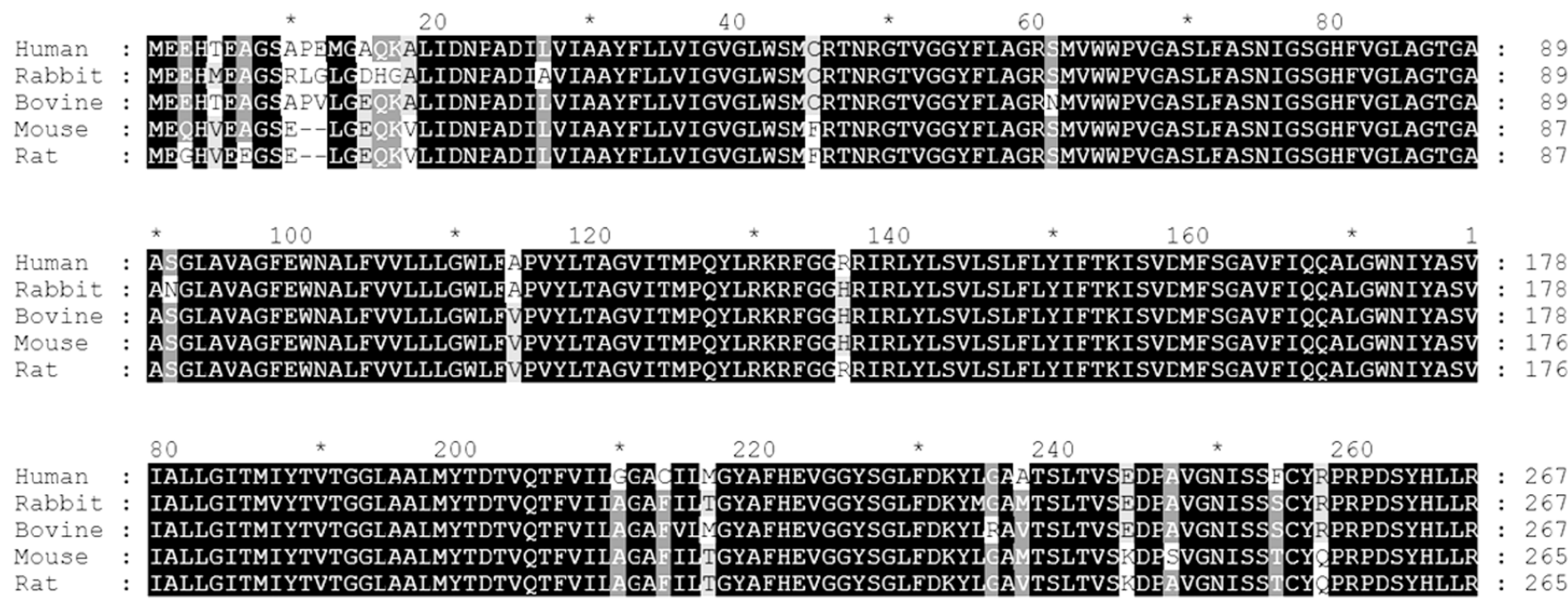

260
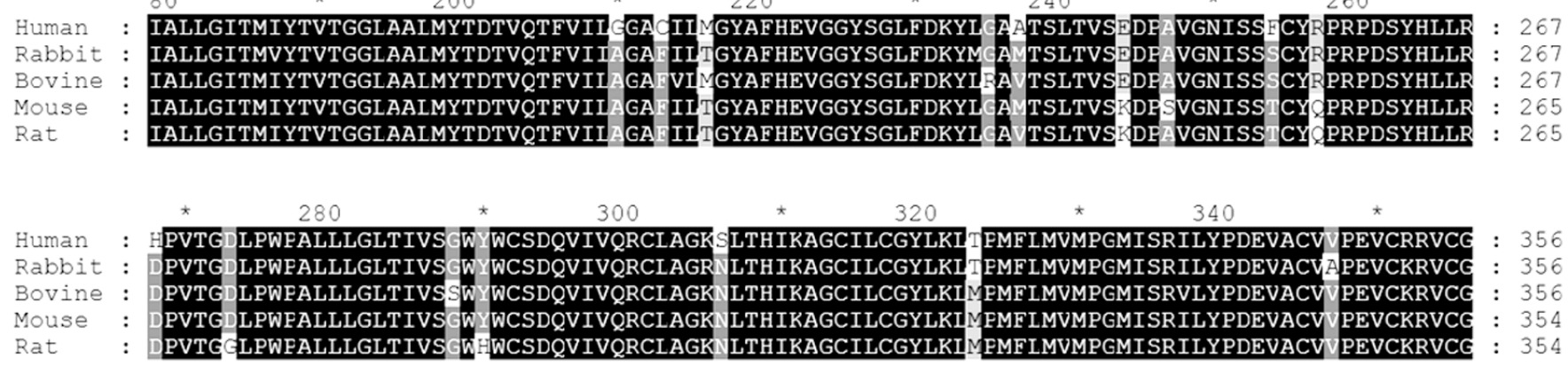

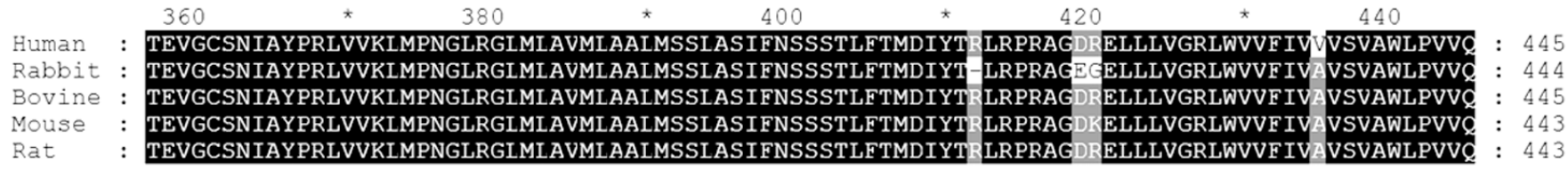
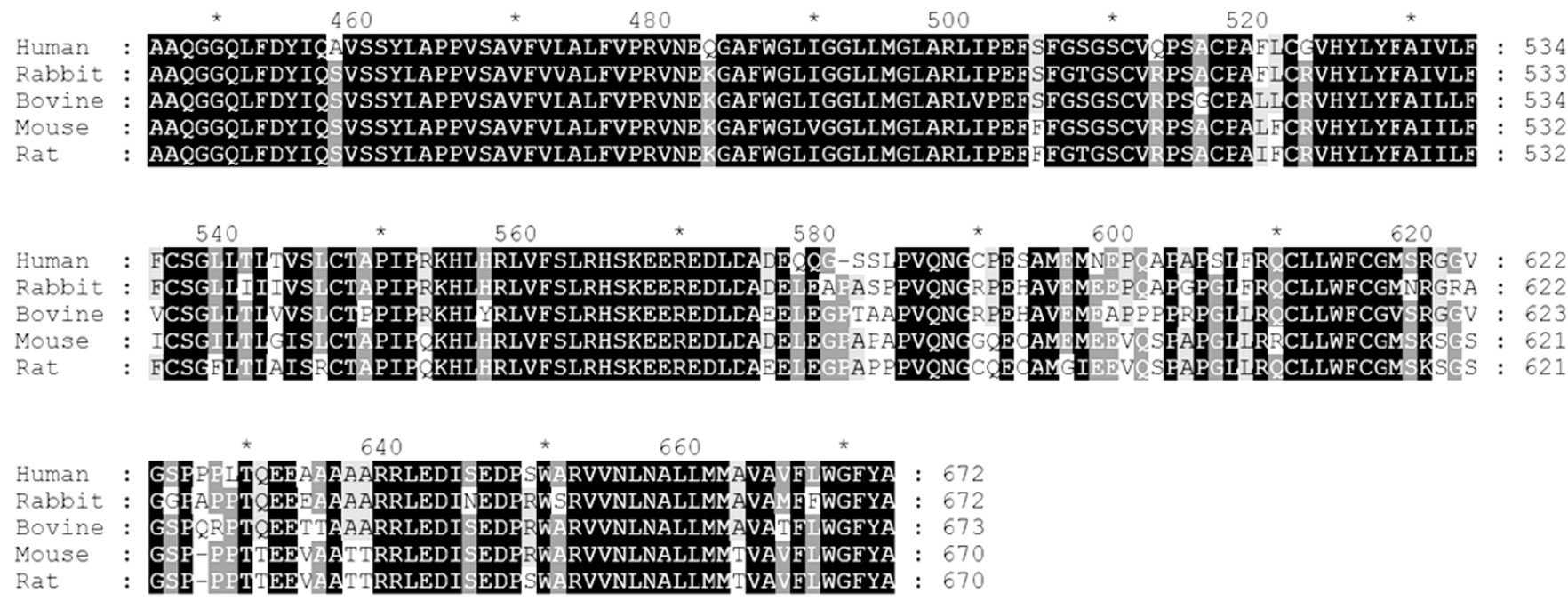

Figure 3. Multiple sequence alignment of the deduced amino acid sequence of bovine SGLT2 with the sequences of human (NM_003041), rabbit (M84020), mouse (NM_133254), and rat (NM_022590) SGLT2. The alignments were performed with the CLUSTAL W program (open gap cost 10). Residues highlighted by black shading represent absolutely conserved amino acid; residues with gray shading indicate 3 or 4 conserved residues at that position.

Similar to bSGLT1 and bSGLT5 (Zhao et al., 2005), the sequence of bSGLT2 contains several conserved sodium:solute symporter family signatures that are char- acteristic of this family. The sodium:solute symporter family signature 1 (NA_SOLUT_SYMP_1; accession number PS00456) is located between amino acid 171 
A

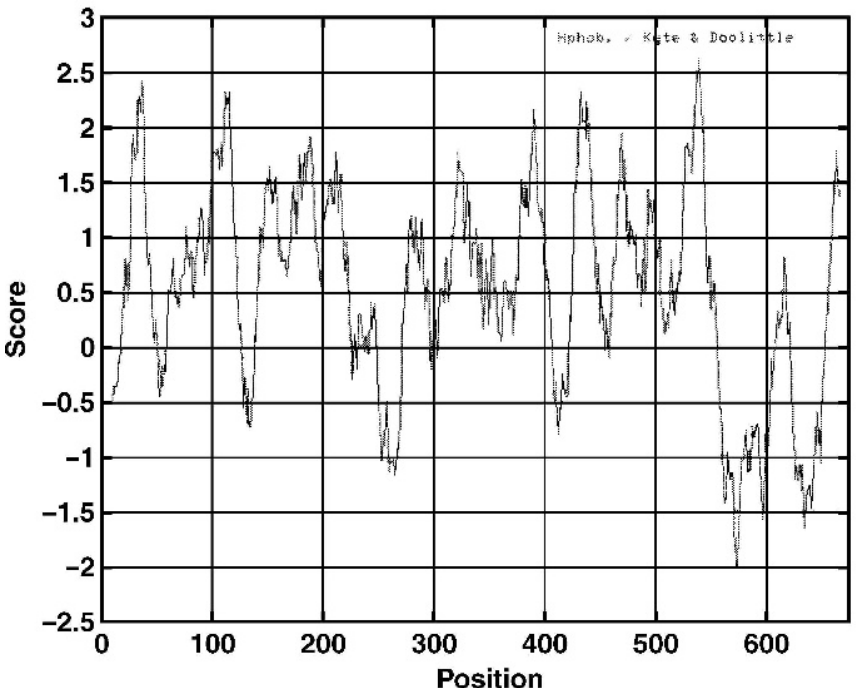

B

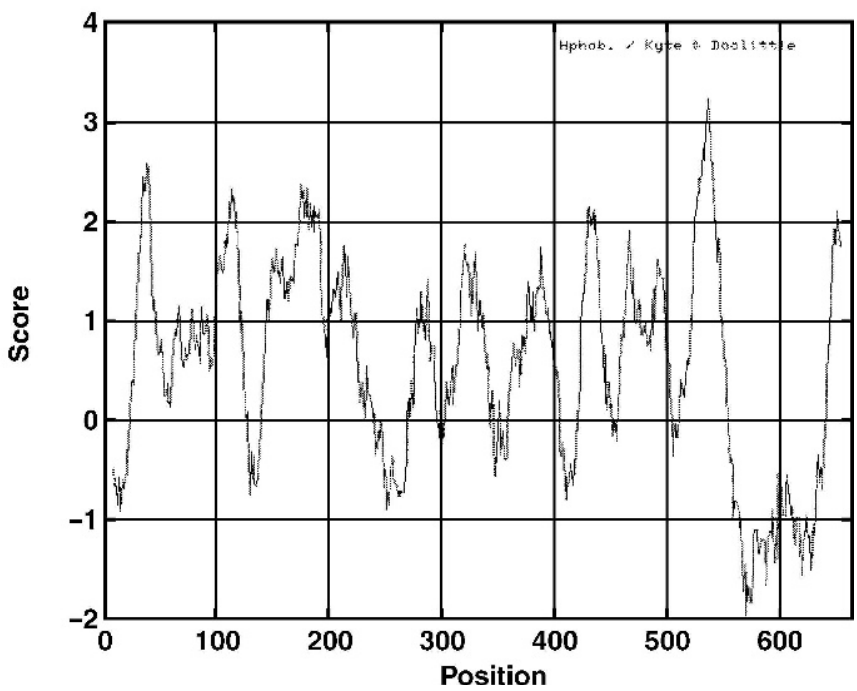

Figure 4. Comparison of hydropathy plots of bovine sodium/glucose cotransporter 2 (SGLT2) (A) and bovine SGLT1 (B). Plots were derived according to the algorithm of Kyte and Doolittle (1982), using a 19-amino acid window.

and 196 (GwnIyasVIALLGItmiYTvtGGLaA). The sodium:solute symporter family signature 2 (NA_SOLUT_SYMP_2; PS00457) is located between amino acids 474 and 494 (ALfvpRvnekGAfwGLIGGIL). In addition, the sequence between amino acids 22 and 506 in bSGLT2 matches the sodium:solute symporter family profile (PS50283).

Scan-Prosite analysis predicted 3 potential N-glycosylation sites positioned at amino acids 250 to 253 (NISS), 306 to 309 (NLTH), and 399 to 402 (NSSS) of bSGLT2 (Figure 1). However, it is unclear whether these sites are actually glycosylated, because the in vitro translated product of bSGLT2 showed only a very minor shift in apparent molecular weight after addition of canine microsomal membranes to the reaction (see below). In addition, numerous protein kinase $\mathrm{C}$ and casein kinase II phosphorylation sites are predicted with a high probability of occurrence. The functional significance of these sites remains to be investigated. It has been reported that the transport activity of SGLT1 can be regulated by phosphorylation (Hirsch et al., 1996). However, the effect of protein kinase A is independent of the presence or absence of consensus phosphorylation sites in SGLT1 (Wright et al., 1997), suggesting that its effects are indirect.

\section{Genomic Organization of Bovine SGLT2}

Our BLAST searches of the recently released bovine genomic database using bSGLT2 cDNA indicated that the current bovine genomic sequence data is incomplete. Three internal regions within bSGLT2 cDNA could not be matched to any genomic sequence (Table 2 ). Therefore, we analyzed the genomic organization of human SGLT2 and compared available bovine data with it (Table 2).

Human SGLT2 cDNA (NM_003041) completely aligned to 2 genomic contig sequences (NT_086679 and NT_010393) of chromosomes 5 (5q31-q23) and 16 (16p12-p11), respectively, indicating that the human genome contains 2 copies of the SGLT2 gene. These 2 copies are identical in sequence. As presented in Table 2 and Figure 5, the human SGLT2 gene spans less than $8 \mathrm{~kb}$ and is composed of 14 exons. Both the $5^{\prime}$ and $3^{\prime}$ splice junctions and the sizes of each exon and intron are listed in Table 2 . In each case, the sequences at the boundaries complied with the canonical AG..GT acceptor/donor splice sites. The size of exons ranged from 72 (exon 2) to $460 \mathrm{bp}$ (exon 14). The introns ranged from 74 to $1410 \mathrm{bp}$.

The currently released bovine genomic data confirmed at least 11 exons of the bovine SGLT2 gene (Table 2). The sizes of these exons as well as their flanking introns are the same or very close to their human counterparts. Only partial bSGLT2 sequences corresponding to human exons 5, 8, and 14 could not be found in the bovine genomic database. Because all available data for the majority of the bovine cDNA show the same genomic organization as human SGLT2, it is very likely that the bovine SGLT2 gene also has 14 exons. However, it is not clear whether the bovine genome also contains 2 copies of the SGLT2 gene. 
Table 2. Nucleotide sequences of intron-exon junctions of the bovine and human SGLT2 genes. ${ }^{1}$

\begin{tabular}{|c|c|c|c|c|c|c|}
\hline \multirow[b]{2}{*}{ Exon \# } & \multirow[b]{2}{*}{ Species } & \multirow[b]{2}{*}{ 3'-Intron } & \multirow[b]{2}{*}{ Exon sequence } & \multirow[b]{2}{*}{$5^{\prime}$ Intron } & \multicolumn{2}{|c|}{ Size (bp) } \\
\hline & & & & & Exon & Intron \\
\hline \multirow[t]{2}{*}{1} & Bovine & $\mathrm{NA}^{2}$ & AGA TCG TGG ...... GGC TTG TGG & gtgagaagct & $140^{3}$ & 548 \\
\hline & Human & NA & GGG GCA GAT ...... GGC TTG TGG & gtgagaagtt & $145^{4}$ & 1410 \\
\hline \multirow[t]{2}{*}{2} & Bovine & caacccceag & TCC ATG TGC ...... TGG TGG CCG & gtgagagagg & 72 & 76 \\
\hline & Human & tgtctcccag & TCC ATG TGC ...... TGG TGG CCG & gtgagacggg & 72 & 74 \\
\hline \multirow[t]{2}{*}{3} & Bovine & ggccacccag & GTC GGG GCC ...... GAG TGG AAT & gtgagtccet & 105 & 1040 \\
\hline & Human & ggccacccag & GTT GGG GCC ...... GAG TGG AAT & gtgaggecet & 105 & 805 \\
\hline \multirow[t]{2}{*}{4} & Bovine & gtgcceacag & GCA CTG TTC ...... AAG ATT TCG & gtaaacatgc & 165 & $?$ \\
\hline & Human & ccccccgtag & GCG CTC TTC ...... AAG ATC TCA & gtgagtgect & 165 & 276 \\
\hline \multirow[t]{2}{*}{5} & Bovine $^{5}$ & $?$ & GTG GAC ATG ...... CTG TGA CAG & $?$ & 106 & $?$ \\
\hline & Human & ctcccccaag & GTG GAC ATG ...... CGG TGA CAG & gtgccagcag & 106 & 1044 \\
\hline \multirow[t]{2}{*}{6} & Bovine & ccggttgcag & GAG GGC TGG ...... TGG GTT ACG & gtacgggctc & 81 & 151 \\
\hline & Human & cctgttgcag & GAG GGC TGG ...... TGG GTT ACG & gtaggggctc & 81 & 129 \\
\hline \multirow[t]{2}{*}{7} & Bovine & gecctcacag & CCT TCC ACG ...... AGC GAC CAG & gtgaggggtc & 230 & $?$ \\
\hline & Human & tgcccggeag & CCT TCC ACG ...... AGC GAC CAG & gtgcgggtat & 230 & 278 \\
\hline \multirow[t]{2}{*}{8} & Bovine $^{5}$ & ? & GTT ATT GTG ...... TTT ACC CGG & gtaacgtctg & 136 & 222 \\
\hline & Human & cetcccgtag & GTC ATC GTG ...... TGT ACC CAG & gtaacatccc & 136 & 209 \\
\hline \multirow[t]{2}{*}{9} & Bovine & cttccegcag & ACG AAG TGG ...... TGC CCA ATG & gtaagggegg & 108 & 127 \\
\hline & Human & tctctggcag & ACG AGG TGG ...... TGC CCA ACG & gtaagggcag & 108 & 131 \\
\hline \multirow[t]{2}{*}{10} & Bovine & ccgeccgcag & GTC TGC GCG ...... AGT AGG ACG & gtgggectgg & 151 & 112 \\
\hline & Human & cggccegcag & GTC TGC GCG ...... GGT GGG ACG & gtgeggectg & 151 & 107 \\
\hline \multirow[t]{2}{*}{11} & Bovine & cccaccgcag & GCT CTG GGT ...... AAC GAG AAG & gtgagcgtgt & 169 & 77 \\
\hline & Human & tccgecgcag & GCT CTG GGT ...... AAT GAG CAG & gtgagcggca & 169 & 74 \\
\hline \multirow[t]{2}{*}{12} & Bovine & ggcccegcag & GGC GCC TTC ...... CGC AAG CAC & gtaatggcag & 216 & 739 \\
\hline & Human & tccttcgcag & GGC GCC TTC ...... AGA AAG CAC & gtgagtggec & 216 & 765 \\
\hline \multirow[t]{2}{*}{13} & Bovine & cettccccag & CTC TAC CGC ...... AGA TGG AGG & gtaaggcact & 130 & 87 \\
\hline & Human & ccetccccag & CTC CAC CGC ...... AGA TGA ATG & gtagggcacc & 127 & 78 \\
\hline \multirow[t]{2}{*}{14} & Bovine $^{5}$ & cttctcccag & CGC CCC CGC ...... TGC CTA GTC & NA & 452 & NA \\
\hline & Human & tttccctcag & AGC CCC AGG ...... TTC CCC TGT & NA & 460 & NA \\
\hline
\end{tabular}

\footnotetext{
${ }^{1}$ Exact sizes of exons and introns and their locations were obtained from the released data of the bovine and human genomic sequences in GenBank (Accession numbers: AAFC01429583; AAFC01221635; AAFC01169134; AAFC01288208; and NT_010393.15).

${ }^{2} \mathrm{NA}=$ Not applicable; $?=$ data not available.

${ }^{3}$ Exact size of exon 1 based on our $5^{\prime}$ RACE data.

${ }^{4}$ Approximate size of human exon 1 based on human SGLT2 sequence (NM_003041).

${ }^{5}$ Part of the genomic sequence for this exon and/or its flanking introns was not available in GenBank. The information listed was based on the size of the human exon.
}

\section{In Vitro Transcription and Translation of bSGLT2 cDNA and Glycosylation of bSGLT2}

Coupled in vitro transcription and translation was done to characterize the bSGLT2 gene product (Figure 6 ). The translation product migrated at an apparent molecular weight of $55 \mathrm{kDa}$, which was very close to the size of in vitro translated rat SGLT2 (You et al., 1995), but considerably smaller than the $73 \mathrm{kDa}$ predicted from the sequence of the open reading frame.
This inconsistency has been observed in all glucose transporter isoforms we have examined, including bGLUT1, bGLUT8, and bSGLT1 (Zhao et al., 2004, 2005) and may be due to initiation of translation at a different site than predicted. Upon addition of canine microsomal membranes to the translation reactions, the apparent size of the translation product increased only slightly, in contrast to the significant increase in bSGLT1 (Figure 6) and rat SGLT2 (You et al., 1995), making it unclear whether bSGLT2 is glycosylated.

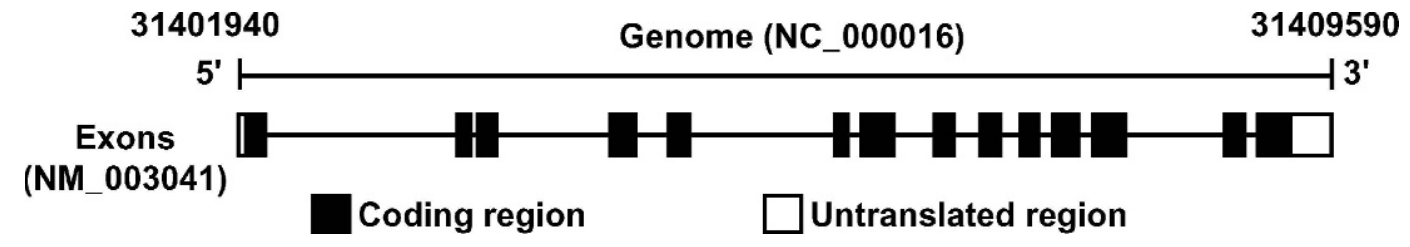

Figure 5. Genomic organization of the human sodium/glucose cotransporter 2 (SGLT2) gene. The figure was drawn based on the human genomic sequence (Accession number NC_000016), human SGLT2 cDNA (NM_003041), and its deduced amino acid sequence (NP_003032). 
Similarly, the functional significance of glycosylation of SGLT is not known. It has been shown that glycosylation does not appear to be required for activity of human SGLT1 (Wright and Turk, 2004).

\section{Distribution of SGLT2 mRNA in Bovine Tissues}

Northern blot analysis using a cDNA probe derived from the 3 ' region of the SGLT2 cDNA detected SGLT2 mRNA predominantly in bovine kidney as a $2.3-\mathrm{kb}$ transcript and at lower levels in all other bovine tissues we examined, including the mammary gland, liver, lung, spleen, intestine, and skeletal muscle, as a 3.0-kb transcript (Figure 7). The expression of SGLT2 mRNA in lactating mammary gland is consistent with the results of our BLAST search of GenBank, using the human SGLT2 cDNA sequence, which allowed us to identify EST clones for SGLT2 (BE481971, 86\% of identity; BF230764, 91\%) in the USDA Beltsville Agricultural Research Center cDNA library of bovine mammary tissues.

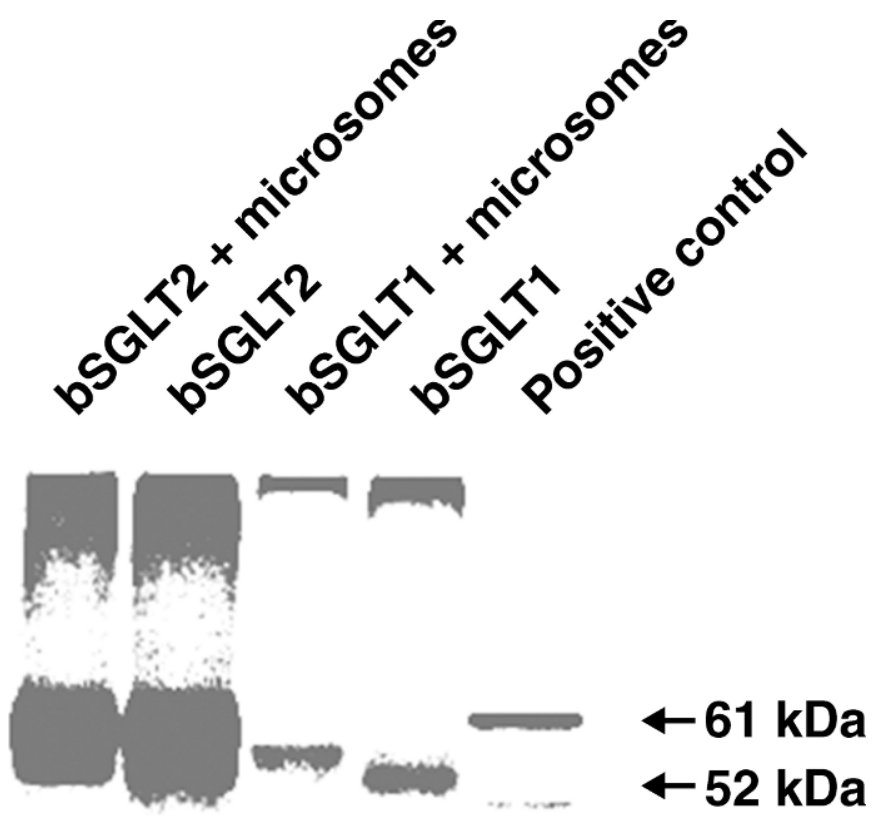

\section{$\begin{array}{lllll}1 & 2 & 3 & 4 & 5\end{array}$}

Figure 6. In vitro transcription and translation of bovine sodium/ glucose cotransporter 2 (SGLT2) and SGLT1 cDNA with or without addition of canine microsomal membranes to the reaction. Firefly luciferase cDNA was used as a positive control. The positions of translation products are indicated.

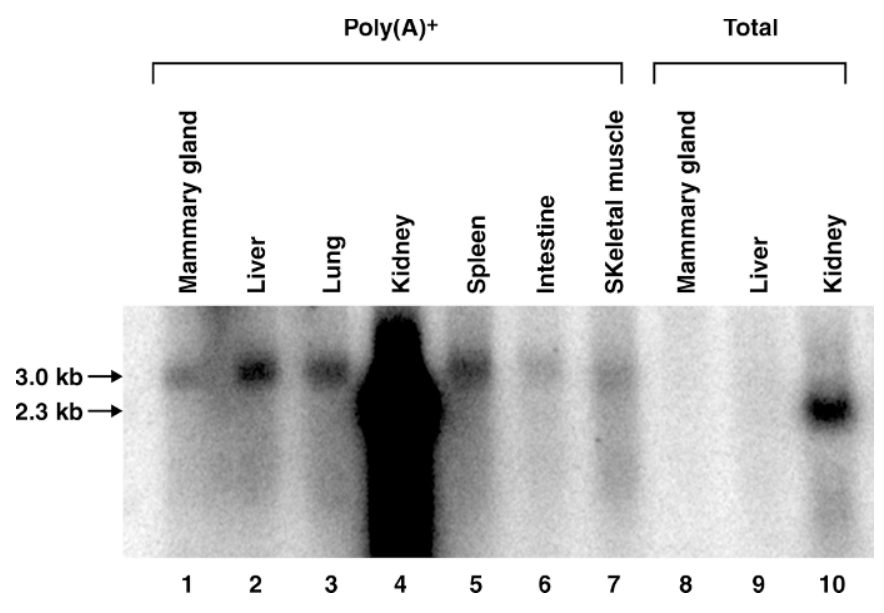

Figure 7. Northern blot analysis of the distribution of bovine sodium/glucose cotransporter 2 (SGLT2) mRNA in bovine tissues. The sizes of the hybridizing transcripts are indicated. The RNA was isolated from 2 lactating cows. The amount of RNA loaded in each lane was $5 \mu \mathrm{g}$ of polyadenylated RNA [poly(A) ${ }^{+}$(lanes 1 to 7 ) or 20 $\mu \mathrm{g}$ of total RNA (lanes 8 to 10).

The relative expression levels of SGLT2 mRNA in bovine tissues are consistent with its reported distribution in human and rat. In those species, SGLT2 is predominantly located on the apical membrane of the S1 and S2 segments of renal proximal convoluted tubules (Wells et al., 1992; Kanai et al., 1994; Wright, 2001), thus it is presumed to be mainly responsible for the reabsorption of D-glucose from the glomerular filtrate. This assumption is further supported by genetic evidence that homozygous and heterozygous nonsense mutations of SGLT2 are associated with autosomal recessive renal glycosuria in human patients (van den Heuvel et al., 2002; Kleta et al., 2004). The expression pattern of SGLT2 mRNA in bovine tissues reported here indicates that SGLT2 may play a similar role in ruminants.

Sodium-dependent glucose transport is believed to be restricted to small intestine and kidney for absorption of glucose and galactose from digesta or for reabsorption of these nutrients from the glomerular filtrate. The physiological significance of SGLT2 expression in other tissues is uncertain. It will be important to confirm the expression of SGLT2 protein in these tissues. However, because members of the sodium/glucose cotransporter family play multifunctional roles, including functioning as uniporters, urea and water channels, and urea and water cotransporters (Wright and Turk, 2004), and because they cotransport multiple substrates along with sodium flux (sugars, inositol, proline, pantothenate, iodide, urea, and undetermined solutes), it will be a challenge to determine the physiological role(s) of these transporters in individual tissues. 


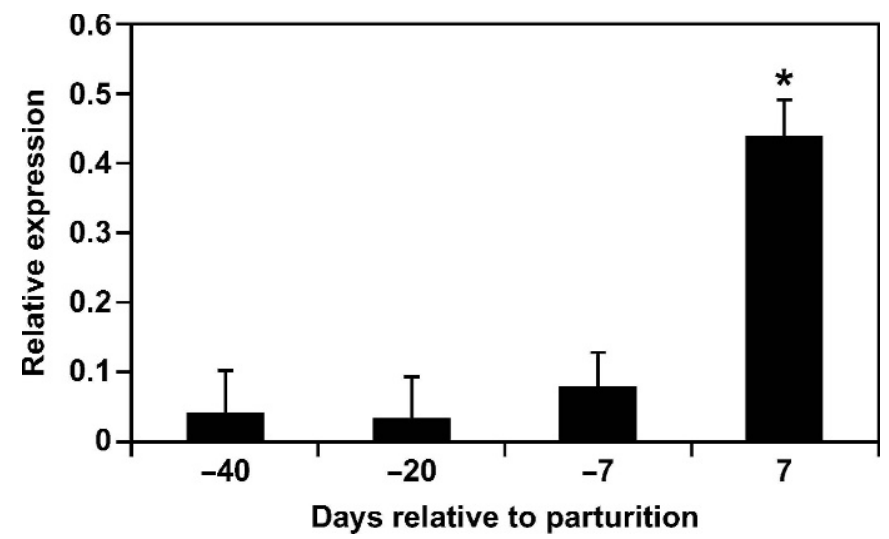

Figure 8. Developmental regulation of sodium/glucose cotransporter 2 (SGLT2) mRNA expression in the bovine mammary gland during pregnancy and lactation by quantitative reverse transcriptionPCR analysis. The relative expression of bSGLT2 was normalized to that of $\beta$-actin. Data shown are means \pm SEM $(n=12)$. The overall effect of developmental stage was significant $(P<0.01)$. Asterisks indicate significant differences relative to the previous time point $(P$ $\leq 0.01)$.

\section{Developmental Regulation of SGLT2 Expression in Bovine Mammary Gland}

As SGLT2 expression in bovine mammary gland was confirmed by our Northern blot analysis, we wanted to determine whether its expression is developmentally regulated. We analyzed the relative expression of the SGLT2 mRNA in mammary tissues from cows at $d-40$, $-20,-7$, and +7 relative to expected calving. The results are shown in Figure 8. Similar to SGLT1 (Zhao et al., 2005), expression of SGLT2 in bovine mammary gland is developmentally regulated during pregnancy and lactation. SGLT2 mRNA levels in bovine mammary gland increased more than 10 -fold $(P<0.01)$ from $\mathrm{d}-40$ to $\mathrm{d}+7$ relative to parturition, a more marked response compared with that of SGLT1 expression, which increased 4-fold over the same interval (Zhao et al., 2005).

Developmental regulation of SGLT2 in bovine mammary gland suggests that it may function in supporting milk synthesis and implies that its expression may be regulated by lactogenic hormones. However, the role it plays in the mammary gland is uncertain. Presence of SGLT2 in the basolateral membrane of mammary epithelial cells to enable active glucose uptake would not be consistent with the large downward concentration gradient across the membrane (Faulkner et al., 1981) and would challenge the concept that glucose is transported into mammary cells solely through a passive process of facilitated diffusion (Xiao and Cant, 2003). It is also possible that SGLT2 may localize to Golgi membranes, and function in the uptake of glucose from cytosol to Golgi apparatus to support lactose syn- thesis, or to apical membranes, where it could be involved in transporting glucose between the alveolar lumen and the cytoplasm of the epithelial cells (Faulkner et al., 1981). Thus, it will be important to determine the cellular and subcellular localization of SGLT2 in mammary tissue to gain insight into its function. In addition, as mentioned above, SGLT have multifunctional roles and may be involved in transporting other substrates in addition to glucose.

\section{CONCLUSIONS}

In this paper, we report the cDNA and the deduced amino acid sequences of bovine SGLT2 and its expression in bovine tissues. The expression pattern of bovine SGLT2 indicates that it may play a major role in glucose reabsorption in kidney. The expression and developmental regulation of bSGLT2 in the mammary gland suggests that it may have a physiological role in milk synthesis. Our data lay the groundwork for future studies aimed at unraveling the functional roles of bSGLT2 in supporting milk production and maintaining glucose homeostasis during lactation.

\section{ACKNOWLEDGMENTS}

The work was supported by the United States Department of Agriculture Hatch grants VT-AS-00808 and VT-AS-00915 (to FQZ) and by the United States-Israel Binational Agricultural Research and Development Fund (BARD) US-3201-01R (to TBM).

\section{REFERENCES}

Faulkner, A., N. Chaiyabutr, M. Peaker, D. T. Carrick, and N. J. Kuhn. 1981. Metabolic significance of milk glucose. J. Dairy Res. 48:51-56.

Hirsch, J. R., D. D. Loo, and E. M. Wright. 1996. Regulation of $\mathrm{Na}^{+} /$ glucose cotransporter expression by protein kinases in Xenopus laevis oocytes. J. Biol. Chem. 271:14740-14746.

Kanai, Y., W. S. Lee, G. You, D. Brown, and M. A. Hediger. 1994. The human kidney low affinity $\mathrm{Na}^{+}$/glucose cotransporter SGLT2. Delineation of the major renal reabsorptive mechanism for Dglucose. J. Clin. Invest. 93:397-404.

Kleta, R., C. Stuart, F. A. Gill, and W. A. Gahl. 2004. Renal glucosuria due to SGLT2 mutations. Mol. Genet. Metab. 82:56-58.

Kyte, J., and R. F. Doolittle. 1982. A simple method for displaying the hydropathic character of a protein. J. Mol. Biol. 157:105-132.

Natalizio, B. J., L. C. Muniz, G. K. Arhin, J. Wilusz, and C. S. Lutz. 2002. Upstream elements present in the $3^{\prime}$-untranslated region of collagen genes influence the processing efficiency of overlapping polyadenylation signals. J. Biol. Chem. 277:42733-42740.

Thompson, J. D., D. G. Higgins, and T. J. Gibson. 1994. CLUSTAL W: Improving the sensitivity of progressive multiple sequence alignment through sequence weighting, positions-specific gap penalties and weight matrix choice. Nucleic Acids Res. 22:4673-4680.

Turk, E., and E. M. Wright. 1997. Membrane topology motifs in the SGLT cotransporter family. J. Membr. Biol. 159:1-20. 
van den Heuvel, L. P., K. Assink, M. Willemsen, and L. Monnens. 2002. Autosomal recessive renal glucosuria attributable to a mutation in the sodium glucose cotransporter (SGLT2). Hum. Genet. 111:544-547.

Wells, R. G., A. M. Pajor, Y. Kanai, E. Turk, E. M. Wright, and M. A. Hediger. 1992. Cloning of a human kidney cDNA with similarity to the sodium-glucose cotransporter. Am. J. Physiol. 263:F459-F465.

Wright, E. M. 2001. Renal Na(+)-glucose cotransporters. Am. J. Physiol. Renal Physiol. 280:F10-F18.

Wright, E. M., J. R. Hirsch, D. D. Loo, and G. A. Zampighi. 1997. Regulation of $\mathrm{Na}^{+} /$glucose cotransporters. J. Exp. Biol. 200:287-293.

Wright, E. M., and E. Turk. 2004. The sodium/glucose cotransport family SLC5. Pflugers Arch. 447:510-518.
Xiao, C., and J. P. Cant. 2003. Glucose transporter in bovine mammary epithelial cells is an asymmetric carrier that exhibits cooperativity and trans-stimulation. Am. J. Physiol., Cell Physiol. 285:C1226-C1234.

You, G., W. S. Lee, E. J. Barros, Y. Kanai, T. L. Huo, S. Khawaja, R. G. Wells, S. K. Nigam, and M. A. Hediger. 1995. Molecular characteristics of $\mathrm{Na}(+)$-coupled glucose transporters in adult and embryonic rat kidney. J. Biol. Chem. 270:29365-29371.

Zhao, F.-Q., P. J. Miller, E. H. Wall, Y.-C. Zheng, B. Dong, M. C. Neville, and T. B. McFadden. 2004. Bovine glucose transporter GLUT8: Cloning, expression, and developmental regulation in mammary gland. Biochim. Biophys. Acta 1680:103-113.

Zhao, F.-Q., Y.-C. Zheng, E. H. Wall, and T. B. McFadden. 2005. Cloning and expression of bovine sodium/glucose cotransporters. J. Dairy Sci. 88:182-194. 